\title{
VISUAL OUTCOME AND INTRAOCULAR PRESSURE CONTROL AFTER CATARACT SURGERY IN LENS INDUCED GLAUCOMA
}

Rani Sujatha, Prashanth C.N, Praveen Kumar Patil.

1. Professor \& HOD, Department of Ophthalmology, Dr. B. R. Ambedkar Medical College.

2. Assistant Professor, Department of Ophthalmology, Dr. B. R. Ambedkar Medical College.

3. Consultant Ophthalmologist \& Eye Surgeon, Department of Ophthalmology, Trichur Eye Hospital \& Diabetic Center.

\section{CORRESPONDING AUTHOR}

\section{Dr. Prashanth C. N.}

\# G-004, Uday residency,

Kempapura Main Road, Hebbal Kempapura,

Hebbal, Bangalore - 560024.

Email-prashanthcndoctor@gmail.com

\section{HOW TO CITE THIS ARTICLE:}

Rani Sujatha, Prashanth C. N, Praveen Kumar Patil. "Visual outcome and intraocular pressure control after cataract surgery in lens induced glaucoma". Journal of Evolution of Medical and Dental Sciences 2013; Vol2, Issue 26, July 1; Page: 4763-4777.

INTRODUCTION: The cataract backlog is estimated to be around 12 million $^{1}$ and annually increasing at an estimated rate of 3.8 million. ${ }^{2}$ Lens induced glaucoma (LIG) is also found to be fairly common in India. ${ }^{3,4}$

Lens induced glaucoma (LIG) due to hyper mature cataracts are an important cause of secondary glaucoma in the developing world. There is an ever-increasing backlog of cataract due to the population explosion, increased life expectancy and low productivity in terms of utilization of the available surgical services. The uptake of eye care services by the rural community has also been suboptimal in countries like India where lens induced glaucoma (LIG) is a common cause of ocular morbidity. ${ }^{5}$

Pre-operative and post-operative morbidity is more in an eye with lens induced glaucoma than that with a simple uncomplicated cataract. Prognosis and visual outcome are also affected to some extent. Hence timely intervention is important in achieving good results.

Each case depending on the mechanism by which glaucoma occurs, has to be managed in a different way. In general, after controlling the intraocular pressure, cataract extraction with IOL implantation should be performed preferably.

The present study was undertaken and aimed at a clinical study of various lens induced glaucoma, their management, post operative visual outcome and intraocular pressure control as practiced in the department of ophthalmology, Dr. B. R. Ambedkar Medical College Hospital, Bangalore.

\section{OBJECTIVES}

a) To study the various modes of presentation in different lens induced glaucoma.

b) To determine the various factors affecting the course of disease under study. 
c) To assess the surgical treatment of lens induced glaucoma.

d) To assess the final visual outcome in different types of lens induced glaucoma.

e) To study the intra ocular pressure control pre and post operatively.

METHODOLOGY: The aim of this study is to study the incidence, management and prognosis of lens induced glaucoma. All the cases studied were admitted and treated at the ophthalmic ward, Dr. B. R. Ambedkar Medical College Hospital. While selecting the cases for the study, special care was taken not to include cases of primary glaucoma with cataract. Informed consent was taken from all the patients.

SOURCE OF DATA: All the 50 patients were those who attended the inpatient department of Dr. B. R. Ambedkar Medical College Hospital and were diagnosed to be having Lens Induced Glaucoma.

INCLUSION CRITERIA: The following criteria were applied to clinically diagnose the various types of lens induced glaucoma:

1. Phacomorphic glaucoma:

- Shallow anterior chamber

- Intumescence of mature cataract

- Raised IOP

2. Phacolytic glaucoma:

- Deep anterior chamber

- Hypermature cataract

- Raised IOP

- Varying degree of aqueous flare and cells and no K. P's.

3. Phacotoxic uveitis/ lens particle glaucoma:

- Hypermature cataract.

- Traumatic rupture of lens capsule or postoperative retained lens matter

- Mild to moderate signs of iridocyclitis, deep anterior chamber.

- Very few or no K. P's

4. Phacoanaphylactic uveitis with secondary glaucoma:

- History of cataract surgery or injury to lens

- Very severe clinical picture of iridocyclitis.

- Large number of mutton fat like K. P's

5. Glaucoma secondary to ectopia lentis:

- Anterior or posterior dislocation

- Lens across pupillary plane.

- Raised IOP.

\section{EXCLUSION CRITERIA:}

1. Cases of primary glaucoma associated with cataract

2. Cases of lens induced glaucoma with significant anterior and posterior segment pathology

3. Aphakic or pseudophakic glaucoma

4. Other associated glaucoma with cataract such as pseudo-exfoliation glaucoma etc.

SAMPLING SIZE AND METHODS: A minimum of 50 cases of lens induced glaucoma were selected based on simple random sampling. Age and sex were not criteria in selecting cases. The study was 


\section{ORIGINAL ARTICLE}

made on patients diagnosed with lens induced glaucoma and admitted to the ophthalmic ward, Dr. B. R. Ambedkar Medical College Hospital, Bangalore during the period of November 2008 to September 2010.

Patient evaluation: All the 50 patients after clinical diagnosis were admitted and a detailed history and examination were done.

Detailed history pertaining to the causation of glaucoma in the affected eye was ascertained. The details of history included the duration and progress of diminution of vision, onset of pain, redness, watering and photophobia in the affected eye and associated symptoms such as headache, nausea and vomiting were taken. Any history of ocular trauma of previous surgery in the eye was ruled out.

The physical examination of all these 50 patients included a thorough examination of their general and systemic conditions. Examination of the globe and adnexa included all the features that help in diagnosing the condition and also the features that may affect the final visual outcome.

In the affected eye vision was recorded and retinal function test was done by assessing the perception of light and projection of rays.

Intraocular pressure was measured using Perkins tonometer \&two readings were taken to establish the final IOP. In cases where there was corneal edema, IOP \& keratometry were measured after reducing the pressure with IV Mannitol.

B-scan was done in the affected eye as the fundus was not visible to rule out posterior segment pathology.

A detailed examination of the other eye was done.

Other routine tests like lacrimal syringing, keratometry \& A scan biometry were done.

MANAGEMENT: The management of the cases included relief from pain and bringing down the raised IOP. Apart from these, subsidence of the uveal inflammation was also a priority. These were achieved through both medical and surgical measures.

Medical management: Medical management consisted of systemic analgesics\& topical medications like mydriatics (after assessing angle of the anterior chamber), topical steroids \& anti-glaucoma drugs.

Oral analgesics given in majority was Tab Diclofenac Sodium; Intramuscular injections was preferred if patient had associated vomiting.

Mydriatic were used in cases with open angle mechanism type of glaucoma. Topical Atropine Sulphate $1 \%$ eye ointment was also used in some patients.

Topical steroid preferred was Prednisolone acetate 1\% eye drops every hourly application which helps to bring down the inflammation. Systemic steroids were preferred in cases of severe iridocyclitis with Tab. Prednisolone $1 \mathrm{gm} / \mathrm{kg}$ body weight/day.

To reduce the IOP, Inj. Mannitol 20\%, 100ml was given IV along with tab Acetazolamide $500 \mathrm{mg}$ stat followed by $250 \mathrm{mg} 6^{\text {th }}$ hourly. This was supplemented with topical beta-blockers like $0.5 \%$ Timolol maleate eye drops twice daily.

Supportive management consisted of anti-emetics such as Inj. Domperidone IV to reduce vomiting. 


\section{ORIGINAL ARTICLE}

PREOPERATIVE MANAGEMENT: Prior to surgery, IOP was recorded in all patients and if the IOP was still raised Inj. Mannitol 20\%, $100 \mathrm{ml}$ IV was given at the rate of 50drops/min over a period of 30 min along with oral Tab. Acetazolamide 500mg stat $250 \mathrm{mg}$ QID maintenance.

Pre-operatively pupils were dilated with combination of Tropicacyl $(0.08 \%)$ and Phenylephrine (5\%) eye drops, instilled every 15 minutes until the pupil dilated. 1 drop of Flurbiprofen $(0.03 \%)$ eye drops were instilled 1 /2hourly 3 times preoperatively to prevent intraoperative miosis.

SURGICAL MANAGEMENT: Following control of IOP, patient underwent Manual Small Incision Cataract Surgery with PCIOL implantation under peribulbar anesthesia. Suitable modifications in the surgical steps were made according to the situation encountered.

POSTOPERATIVE MANAGEMENT: Eye pad was removed next day. Detailed slit lamp evaluation was done and looked for any post-operative complications. Vision with pin hole was tested on the first day.

All the patients were given topical antibiotic; steroid drops to be instilled 8-10 times daily for a period of 6 weeks on tapering dose.

Patients were followed up after 1, 2, 4 and 6 weeks and at each visit a detailed slit lamp examination, tonometry and BCVA were recorded.

The above data were compiled using a standardized proforma and at the end of the study the data was analyzed statistically using the Chi-Square method and the Probability value. The statistical analysis was done using the 'MINITAB for Windows' software.

\section{RESULTS}

\section{TABLE 1: Age distribution:}

\begin{tabular}{|l|l|l|l|l|}
\hline Age in years & $\mathbf{4 5 - 5 5}$ & $\mathbf{5 6 - 6 5}$ & $\mathbf{6 6 - 7 5}$ & $\mathbf{> 7 5}$ \\
\hline Males & 3 & 7 & 9 & 2 \\
\hline Females & 7 & 9 & 11 & 2 \\
\hline Total & 10 & 16 & 20 & 4 \\
\hline
\end{tabular}

$\chi^{2}=9.632$, p-value $=0.278$

Among the 50 cases that were included in the study, majority of the cases were in the age group of 66- 75 years (40\%). The youngest case in the study group was 52 years old while the oldest was 81 year old.

TABLE 2: SEX DISTRIBUTION

\begin{tabular}{|l|l|l|}
\hline Sex & Number of cases. & Percentage \\
\hline Male & 21 & 42 \\
\hline Female & 29 & 58 \\
\hline Total & 50 & 100 \\
\hline
\end{tabular}

The incidence of lens induced glaucoma was slightly higher in females (58\%) than males (42\%). 


\section{ORIGINAL ARTICLE}

\section{TABLE 3: EYE AFFECTED}

\begin{tabular}{|l|l|l|}
\hline Eye affected & Number of cases & Percentage \\
\hline RE & 19 & 38 \\
\hline LE & 31 & 62 \\
\hline
\end{tabular}

$\chi^{2}=19.321$ and $\mathrm{p}$-value $=0.9$

In 19 cases (38\%), right eye was affected while in 31 cases (62\%) left eye was affected.

TABLE 4: DURATION OF SYMPTOMS

\begin{tabular}{|l|l|l|}
\hline Duration(weeks) & No. Of patients & Percentage \\
\hline$<1$ week & 29 & 58 \\
\hline $1.1-2$ weeks & 11 & 22 \\
\hline$>2.1$ weeks & 10 & 20 \\
\hline Total & 50 & 100 \\
\hline
\end{tabular}

Out of the 50 cases, 29 cases (58\%) presented within the first week and 11 cases (22\%) within $2^{\text {nd }}$ week and rest presented within 3 weeks after development of symptoms.

Table 5: AETIOLOGICAL DIAGNOSIS

\begin{tabular}{|l|l|l|}
\hline AETIOLOGICAL DIAGNOSIS & NO. OF PATIENTS & PERCENTAGE \\
\hline PHACOMORPHIC GLAUCOMA & 34 & 68 \\
\hline PHACOLYTIC GLAUCOMA & 12 & 24 \\
\hline LENS PARTICLE GLAUCOMA & 02 & 04 \\
\hline PHACOANAPHYLACTIC GLAUCOMA & 00 & 00 \\
\hline SUBLUXATION/DISLOCATION & 02 & 04 \\
\hline
\end{tabular}

$\chi^{2}=15.007$ and ' $\mathrm{p}$ '= 0.001

Out of 50 cases, 34 cases (68\%) were diagnosed as phacomorphic glaucoma (PM), 12 cases (24\%) were of phacolytic glaucoma (PL), 2 each (4\%) were phacotoxic glaucoma (PT) and glaucoma secondary to subluxation/dislocation(S/D) of lens.

TABLE 6: CONDITION OF THE OTHER EYE 
ORIGINAL ARTICLE

\begin{tabular}{|l|l|l|}
\hline Condition of other eye & Number Of cases & Percentage \\
\hline Normal & 01 & 02 \\
\hline Immature/Mature cataract & 21 & 42 \\
\hline Pseudophakia & 22 & 44 \\
\hline Aphakia & 06 & 12 \\
\hline
\end{tabular}

$\chi^{2}=39.566$ and ' $\mathrm{p}$ '= 0.1

In the 50 cases, 22 cases (44\%) presented with pseudophakia, 21 cases (42\%) presented with combined immature and mature cataract, 6 cases $(12 \%)$ were aphakic and normal crystalline lens was found in one case $(2 \%)$.

TABLE 7: VISION IN OTHER EYE

\begin{tabular}{|l|l|l|}
\hline BCVA & Number of cases & Percentage \\
\hline $6 / 6-6 / 12$ & 14 & 28 \\
\hline $6 / 18-6 / 60$ & 16 & 32 \\
\hline$>6 / 60$ & 20 & 40 \\
\hline
\end{tabular}

$\chi^{2}=14.980$ and $\mathrm{p}$-value $=0.2$

Overall, 30 cases $(60 \%)$ had fairly good vision in the other eye while the remaining 20 cases $(40 \%)$ had poor vision in the other eye.

TABLE 8: Pre - Operative IOP

\begin{tabular}{|l|l|l|l|}
\hline & $\mathbf{2 2 - 3 0 \mathbf { m m }} \mathbf{~ h g}$ & $\mathbf{3 0 - 4 0 \mathbf { m m h }}$ & $\mathbf{3} \mathbf{4 0 \mathbf { m m }} \mathbf{~ h g}$ \\
\hline PHACOMORPHIC & 02 & 15 & 17 \\
\hline PHACOLYTIC & 01 & 04 & 07 \\
\hline LENS PARTICLE & - & 01 & 01 \\
\hline PHACOANAPHYLACTIC & - & - & - \\
\hline DISPLACED LENS & - & - & 02 \\
\hline TOTAL & $03(6 \%)$ & $20(40 \%)$ & $27(54 \%)$ \\
\hline
\end{tabular}

$\chi^{2}=8.761, \mathrm{p}=0.1$

A total of 3 cases $(06 \%)$ presented with IOP less than $30 \mathrm{~mm} \mathrm{Hg}, 20$ cases (40\%) with IOP between $30-40 \mathrm{~mm} \mathrm{Hg}$. Rest of the cases presented with IOP more than $40 \mathrm{~mm} \mathrm{Hg} \mathrm{(54 \% ).} \mathrm{The}$ highest pressure recorded during the study was $78.00 \mathrm{~mm} \mathrm{Hg}$. 


\section{ORIGINAL ARTICLE}

\section{TABLE 9: PCR with VL}

\begin{tabular}{|l|l|l|}
\hline PCR with VL & Number of cases & Percentage \\
\hline Phacomorphic & 03 & 06 \\
\hline Phacolytic & 02 & 04 \\
\hline Phacotoxic & 01 & 02 \\
\hline Phacoanaphylactic & - & - \\
\hline Subluxation/ dislocation & - & - \\
\hline Total & 06 & 12 \\
\hline
\end{tabular}

$\chi^{2}=7.053$ AND ' $\mathrm{p}$ '= 0.1

Out of the 50 cases operated, intra-operative complication was noted in 6 cases $(12 \%)$ in the form of PCR with Vitreous Loss.

The incidence of PCR with VL in phacomorphic glaucoma in this study was $6 \%$ whereas in phacolytic glaucoma it was noticed to be around $4 \%$

TABLE 10: AC REACTION

\begin{tabular}{|l|l|l|}
\hline AC reaction & Number of cases & Percentage \\
\hline Phacomorphic & 03 & 8.8 \\
\hline Phacolytic & 06 & 50 \\
\hline Phacotoxic & 02 & 100 \\
\hline Phacoanaphylactic & - & - \\
\hline Subluxation/ dislocation & - & - \\
\hline Total & 11 & 22 \\
\hline
\end{tabular}

$\chi^{2}=55.811$ and ' $\mathrm{p}$ '= 0.1

On the first post-operative day, AC reaction in the form of flare, cells, exudative membrane or hypopyon was found in 11 cases $(22.00 \%)$ out of the 50 cases operated. Six of these cases (54.54\%) were of phacolytic glaucoma. 3 cases (27.27\%) were of phacomorphic glaucoma and 2 cases (18.18\%) were of phacotoxic glaucoma.

TABLE 11: DURATION OF SYMPTOMS AND FINAL BCVA

\begin{tabular}{|l|l|l|l|}
\hline & $\mathbf{6 / 6 - 6 / 1 2 ( \% )}$ & $\mathbf{6 / 1 8}-\mathbf{6 / 6 0}(\%)$ & $>\mathbf{6 / 6 0}(\%)$ \\
\hline$<1$ week & $18(36)$ & $07(14)$ & $04(08)$ \\
\hline 1- 2weeks & $04(08)$ & $05(10)$ & $02(04)$ \\
\hline$>$ 2 weeks & 00 & $05(10)$ & $05(10)$ \\
\hline TOTAL & $22(44)$ & $17(34)$ & $11(22)$ \\
\hline
\end{tabular}

$\chi^{2}=19.533$ and ' $\mathrm{p}$ '= 0.001

Out of 29 cases (58\%) who presented within 1 week, 18 cases $(62.06 \%)$ had good BCVA while 7 cases (24.13\%) had useful vision and remaining 4 cases(13.79\%). 11 cases (22\%) presented between 1- 2weeks in which 4 cases (36.36\%) had good BCVA, 5 cases (45.45\%) had useful vision 
while 5 cases (45.45\%) had poor vision. In 10 cases (20\%) who presented later than 2 weeks, 5 cases $(50 \%)$ had useful vision and 5 cases $(50 \%)$ had poor vision.

TABLE 12: PRE-OP IOP AND FINAL BCVA

\begin{tabular}{|l|l|l|l|l|}
\hline IOP(in mm Hg) & $\mathbf{6 / 6 - 6 / 1 2 ( \% )}$ & $\mathbf{6 / 1 8 - 6 / 6 0 ( \% )}$ & $\mathbf{> 6 / 6 0 ( \% )}$ & TOTAL \\
\hline $\mathbf{2 2 - 3 0}$ & $02(4)$ & 0 & $01(2)$ & $03(6)$ \\
\hline $\mathbf{3 0 - 4 0}$ & $10(20)$ & $06(12)$ & $04(8)$ & $20(40)$ \\
\hline $\mathbf{> 4 0}$ & $10(20)$ & $11(22)$ & $06(12)$ & $27(54)$ \\
\hline
\end{tabular}

$\chi^{2}=17.956$ and ' $\mathrm{p}$ '= 0.04

Out of 3 cases who presented with IOP less than $30 \mathrm{~mm} \mathrm{Hg}, 2$ cases $(66.66 \%)$ had a good BCVA at the end of 6 weeks; remaining 1 case (33.33\%) had poor vision. In 20 cases that presented with IOP between $30-40 \mathrm{~mm} \mathrm{Hg}, 10$ cases (50\%) had good visual acuity while 6 cases (30\%) had fairly useful vision and 4 cases (20\%) had poor vision.

Out of the 27 cases that presented with IOP more than $40 \mathrm{~mm} \mathrm{Hg}, 10$ cases $(37.03 \%)$ had good vision, 11 cases (40.74\%) had fairly useful vision and remaining 6 cases $(22.22 \%)$ cases had poor vision.

DISCUSSION: Even though awareness of cataract \& its surgical treatment is ever increasing in our society, lens induced glaucoma continues to occur more often than not in India.

This longitudinal study attempts to analyze the different types of lens induced glaucoma, the associated risk factors and their consequences on post-operative visual acuity \& intra ocular pressure following small incision cataract surgery.

This study included 50 patients with different lens induced glaucoma. The patients were followed up from the time of admission and surgery till 6 weeks post-operatively and the factors influencing the final visual outcome were analyzed.

Statistically Chi-Square test $\left(\chi^{2}\right)$ and Probability values(p) were used and $p$-value $<0.05$ was considered statistically significant.

AGE DISTRIBUTION: In our study, age range was 52 to 81 years with a mean age of $59.57 \pm 7.96$ years. Highest number of cases occurred in the age group 66-75years (40\%). Lahan study has found occurrence of LIG in the age range of 40 to 80 years and highest between 60 to 69 years (43.1\%) age group, indicating that the lens-induced glaucoma are a condition of old age. ${ }^{6}$

Here $\chi^{2}=9.632$, $p$-value $=0.278$ showing that no significant association existed between the age group of patients and the disease under study.

SEX DISTRIBUTION: In this study, females seemed to have an increased risk of having LIG compared to males with ratio of $1.7: 1$. It is also found that the majority $(77.78 \%)$ of cases occurred among lower socio economic strata in the society. A study done at Madurai in 1994, found marginally significant increased risk of having these glaucoma in females $(p=0.05) .{ }^{7}$ 


\section{ORIGINAL ARTICLE}

Lahan study has reported female to male ratio of 1.7:1.6 Though it is possible that these entities are more common in females because of socio - economic constraints, we also have to consider the fact that the prevalence of cataract itself is more common is females than males.

This finding was consistent with data from the Punjab study in India ${ }^{8}$ and from the Matlab study in Bangladesh. ${ }^{9}$

SOCIO-ECONOMIC BACKGROUND: All the patients who presented with LIG were from rural areas and of poor socioeconomic background. Most of the patients were daily wage workers. This might be one of the causes for late presentation with complications of cataract.

LATERALITY: All cases of lens induced glaucoma studied were unilateral. In 31 cases (62\%) left was affected while in 19 cases (38\%) right eye was affected.

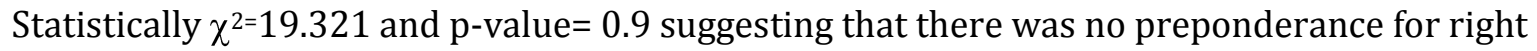
or left eye.

DURATION OF SYMPTOMS: Good visual acuity achieved, in cases presented within 1 week $(62.06 \%)$ was more than the cases presented beyond 2 weeks $(12.5 \%)$, whereas poor visual acuity of less than 6/60 was more in cases presented beyond 2 weeks (50\%). In this study, duration of symptoms had a linear relation with best-corrected visual acuity at final follow up. More the delay in presentation, poorer was the visual outcome, which was both clinically and statistically significant $(\mathrm{p}=0.001)$.

Majority of the cases (58\%) presented with pain and redness within the first week after developing the symptoms. The rest of 21 cases (42\%) presented after 1 week. The above data shows that most patients who neglected the loss of vision presented within a week of developing pain and redness.

STATUS OF OTHER EYE: In the 50 cases, 22 cases (44\%) presented with pseudophakia, 21 cases $(42 \%)$ presented with either immature or mature cataract, 1 case $(2 \%)$ had a normal crystalline lens in the other eye while a further 6 cases $(12 \%)$ were aphakic.

Though clinically significant but not statistically, $\left(\chi^{2}=39.566\right.$ and $p$-value $\left.=0.1\right)$ indicating that the patients having pseudophakia or normal crystalline lens or aphakic in the other eye formed a major bulk of this study.

Overall, 30 cases $(60 \%)$ had fairly good vision in the other eye while the remaining 20 cases $(40 \%)$ had poor vision in the other eye.

Also $\chi^{2}=14.980$, $p$-value $=0.002$ for patients having good vision in other eye indicating the significant association between good vision in other eye and developing LIG.

As a result of having fairly useful vision in the other eye, most of these patients neglected the other eye till they developed complication in the affected eye.

This emphasizes on the importance of early treatment of other eye in patients with bilateral cataract.

INTERPRETATION: In this study series, it is observed that the most frequent type of LIG was PMG (68\%) followed by PLG (24\%), similar occurrence was noted by Lahan study $(72 \%)^{6}$ and Madurai study $(52.7 \%)^{7}$ Occurrences of various lens induced glaucoma in the above studies shows 


\section{ORIGINAL ARTICLE}

variations. Nevertheless, phacomorphic glaucoma has been the most frequent and commonest among all the studies including our study.

In our study, none of PMG occurred below 50 years of age, showing that phacomorphic glaucoma is a disease of old age with preponderance in 50-60 years age group, and the finding was consistent with the 1991 Delhi study. ${ }^{10}$

This is perhaps because of insidious onset, lack of medical awareness and limited resources in developing countries.

While lens particle glaucoma and glaucoma secondary to subluxation/dislocation constituted the remaining $8 \%$, no cases of phacoanaphylactic glaucoma were come across indicating the refinement of cataract surgery.

Phacomorphic and Phacolytic glaucoma formed the main bulk of cases constituting $92 \%$ of cases. This emphasizes the importance of early detection and treatment of cataract.

VISUAL ACUITY: In the 50 cases under study, $94 \%$ cases presented with only perception of light, $4 \%$ with hand movements only and $2 \%$ with counting fingers close to face.

Post operative VA of $6 / 12$ or better was taken as good visual acuity and less than $6 / 60$ as poor visual outcome.

In our study, VA of 6/12 or better is slightly higher (44\%) than Lahan study series $(31.40 \%)$. There were a significant proportion of cases that had poor vision, with visual acuity less than $6 / 60$ $(22 \%)$ similar to Lahan study ${ }^{6}(21.0 \%)$. Thus, it was noticed in our study that higher percentage of cases have achieved good visual recovery and almost equal percentage of cases have poor visual outcome when compared to Lahan study series. ${ }^{6}$

In our study, VA of $6 / 12$ or better was low (44\%) and poor vision of less than 6/60 was higher (22\%) compared to Madurai study, with $59.13 \%$ and $11.82 \%$ respectively. ${ }^{7}$

In our study, good visual acuity achieved by cases with phacolytic glaucoma (53.33\%) was more than phacomorphic glaucoma (37.04\%). This difference was clinically significant but statistically not significant ( $p>0.05$ ). Poor outcome of less than 6/60 showed no significant difference between PLG (26.67\%) and PMG (29.63\%). The 1994 Madurai study series found no statistical difference between the two groups on the final post-operative visual recovery $(p=0.68) .{ }^{7}$

INTRAOCULAR PRESSURE: In an effort to study whether the mechanism of secondary glaucoma has any bearing on IOP, the following observations were made.

Clinically, significant proportion of cases with IOP at presentation less than $30 \mathrm{mmHg}(67 \%)$ achieved good visual acuity, than cases with IOP more than $40 \mathrm{~mm} \mathrm{Hg} \mathrm{(20 \% ),} \mathrm{whereas} \mathrm{no} \mathrm{significant}$ difference was found for poor outcome. The correlation between height of IOP and visual outcome was, clinically significant but statistically not significant ( $p>0.05$ ). Madurai study also had found no statistically significant association between the level of preoperative IOP and final visual acuity. ${ }^{7}$

The IOP at the last follow up was reduced to normal limits $(16.44 \pm 6.54 \mathrm{~mm} \mathrm{Hg})$. This indicates that in lens induced glaucoma IOP should be reduced by medical line of treatment preoperatively to as normal as possible, so as to achieve stable IOP post operatively with no further anti-glaucoma medications.

It is found that the IOP tends to be higher with the delay in presentation beyond 2 weeks than the duration of presentation of less than 2 weeks. Though mean IOP at last follow-up was 


\section{ORIGINAL ARTICLE}

normal (16.44 $\pm 6.54 \mathrm{~mm} \mathrm{Hg}$ ), cases with delay in presentation between 2 to 4 weeks and more than 30 days tend to be on higher end of normal (18.44 $\pm 6.98 \mathrm{~mm} \mathrm{Hg}$ and $18.67 \pm 12.48 \mathrm{~mm} \mathrm{Hg}$ ). Thus delay of more than 2 weeks in presentation would result in higher IOP, especially if the delay is beyond 30 days, which is clinically significant.

There was no significant difference in IOP among LIG sub groups both clinically and statistically. The highest pressure recorded was $78.00 \mathrm{~mm} \mathrm{Hg}$ in the case with phacomorphic glaucoma.

INTRAOPERATIVE COMPLICATIONS: All the cases were treated surgically with small incision cataract surgery with posterior chamber IOL implantation except in 2 cases with traumatic posterior subluxation of lens where scleral fixated IOL was placed.

Out of the 50 cases operated, intraoperative complication was noted in 06 cases $(12 \%)$ in the form of PCR with Vitreous Loss.

PCR with VL occurred in 06 cases out of which, 3 cases (50\%) were phacomorphic glaucoma while 2 cases (33.33\%) were phacolytic glaucoma and one case $(16.66 \%)$ was phacotoxic glaucoma. Five cases were managed with placing PCIOL in sulcus, 1 case was managed by placing ACIOL. With $\chi^{2}=7.053$ and $p$-value $=0.1$, the incidence of PCR with VL was clinically significant but not statistically in the patients with lens-induced glaucoma as compared to normal population.

The above data shows the incidence of PCR during cataract surgery to be $12 \%$ in cases of lens induced glaucoma.

POSTOPERATIVE COMPLICATIONS: On the $1^{\text {st }}$ postoperative day AC reaction in the form of flare, cells, exudative membrane or hypopyon was noticed in 11cases $(22.00 \%)$ out of 50 cases that were operated. Out of the 11 cases, 6 cases (54.54\%) were of phacolytic glaucoma, 3 cases $(27.27 \%)$ were of phacomorphic glaucoma and 2 cases $(18.18 \%)$ of lens particle glaucoma.

With $\chi^{2}=55.811$ and $p$-value $=0.1$ the incidence of AC reaction was clinically significant but not statistically significant in the study population than normal population.

AC chamber reaction was treated with topical steroids, cycloplegic and systemic steroids. Majority of the patients recovered within 1 week with the treatment and all the 11 cases did not show any AC reaction at the end of 2 weeks follow-up period.

CONCLUSION: A clinical study and management of 50 cases of lens induced glaucoma was undertaken with a view to establish the definite role played by the lens in the causation of glaucoma, which is indirectly confirmed by the disappearance of glaucoma on removal of the lens. The following observations were made at the end of the study.

- There was no influence of sex, religion or occupation on the incidence or occurrence of lens induced glaucoma.

- Phacomorphic glaucoma is the most common type of Lens Induced Glaucoma accounting for $68 \%$ of the cases followed by phacolytic glaucoma with $24 \%$ of cases.

- Majority of the patients had good vision in the other eye as a result of which they neglected the affected eye till they developed LIG leading to pain during the time of presentation. Hence the importance to advice the patients regarding the early surgical treatment of cataract before they develop complications. 


\section{ORIGINAL ARTICLE}

- Medical line of management had to be initiated before taking the patients to surgery to reduce the IOP and to relieve the symptoms. However medical line of management was temporary and surgery in the form of lens extraction was the definitive treatment.

- The incidence of intra-operative PCR with VL was more as compared to normal population.

- The incidence of postoperative uveitis was more in LIG when compared to the normal population and more so if associated with intra-operative complications.

- The visual recovery was fairly good in all those cases that have undergone treatment quite early. Also the higher the IOP pre-operatively, the prognosis for good vision following surgery is poor. A sustained rise of pressure for a long time was a bad prognostic factor for post-operative development of good vision.

- It is to be stressed upon, imparting health education and creating awareness regarding cataract and its implications among the rural community, ophthalmic assistants and peripheral health workers.

\section{BIBLIOGRAPHY}

1. Ministry of Health and Family Welfare: Problem of blindness in India. In: Status of National Program for Control of Blindness (NPCB). Government of India, New Delhi 1993:2.

2. Minassian DC, Mehra U. 3.8 million blinded by cataract each year: projection from the first epidemiological study of incidence of cataract in India. Br J Ophthalmol 74:341- 343, 1990

3. Sharma RG, Verma GL, Singhal B. A direct evaluation of Scheie's operation with sclerectomy along with lens extraction in lens induced glaucoma. Ind J Ophthalmol 31:639-641, 1983.

4. Jain IS, Gupta A, Dogra MR, Gangwar DN, Dhir SP. Phacomorphic glaucoma Management and visual prognosis. Ind J Ophthalmol 31:648-653, 1983.

5. Fletcher A, Thulasiraj RD, et al. Low uptake of eye services in rural India: A challenge for programs of blindness prevention. Arch Ophthalmol. 1999; 117:1393-1399.

6. Pradhan. D, Hennig. A, Kumar. J, et al., "A prospective study of 413 cases of LIG in Nepal”. Indian journal of Ophthalmology. 2001; 49(3): 204

7. Prajna NV, Ramakrishnan R, Krishnadas R, Manoharan N. Lens induced glaucoma-results and risk factors for final visual acuity. Indian Journal of Ophthalmology1996; 44 (3):149-55.

8. Chatterjee A, Milton R, Thyle S. Prevalence and aetiology of cataract in Punjab. Br J Ophthalmol. 1982; 66:35-62.

9. Khan M, Hoque M, Khan MR. Prevalence and causes of blindness in rural Bangladesh. Indian J Med Res. 1985; 82:257.

10. Angra SK, Pradha R, Garg SP. Cataract induced glaucoma - an insight into management. Indian Journal of Ophthalmology 1991; 39(3): 97-101 


\section{ORIGINAL ARTICLE}

\section{FIG 1: PHACOMORPHIC GLAUCOMA}

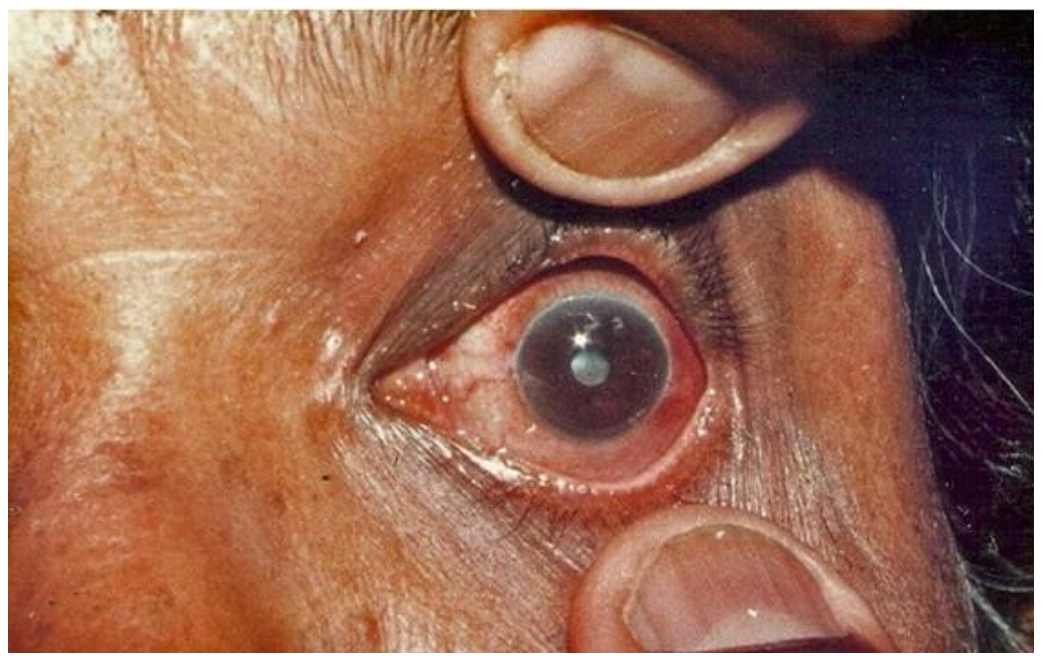

FIG 2: PHACOLYTIC GLAUCOMA

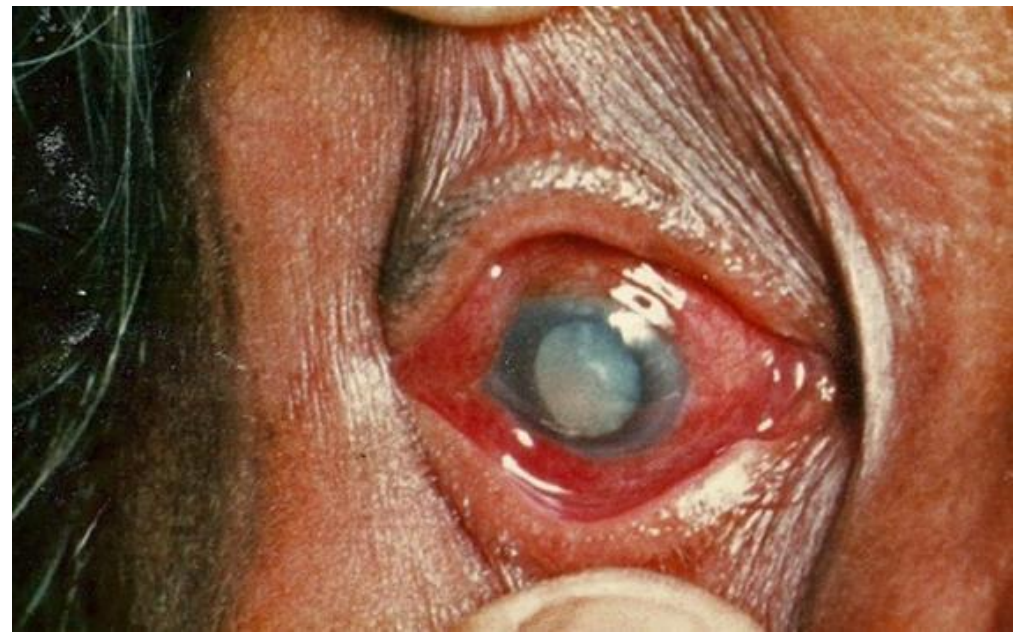

FIG 3: LENS PARTICLE GLAUCOMA

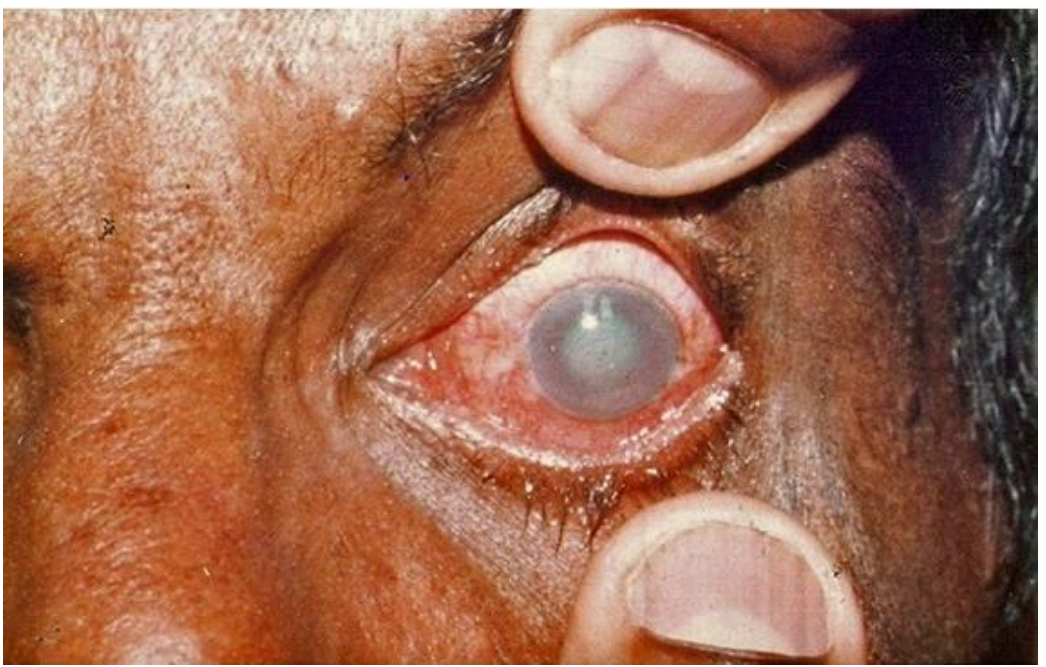




\section{ORIGINAL ARTICLE}

\section{FIG 4: TRAUMATIC ANTERIOR DISLOCATION OF LENS WITH PUPILLARY BLOCK GLAUCOMA}

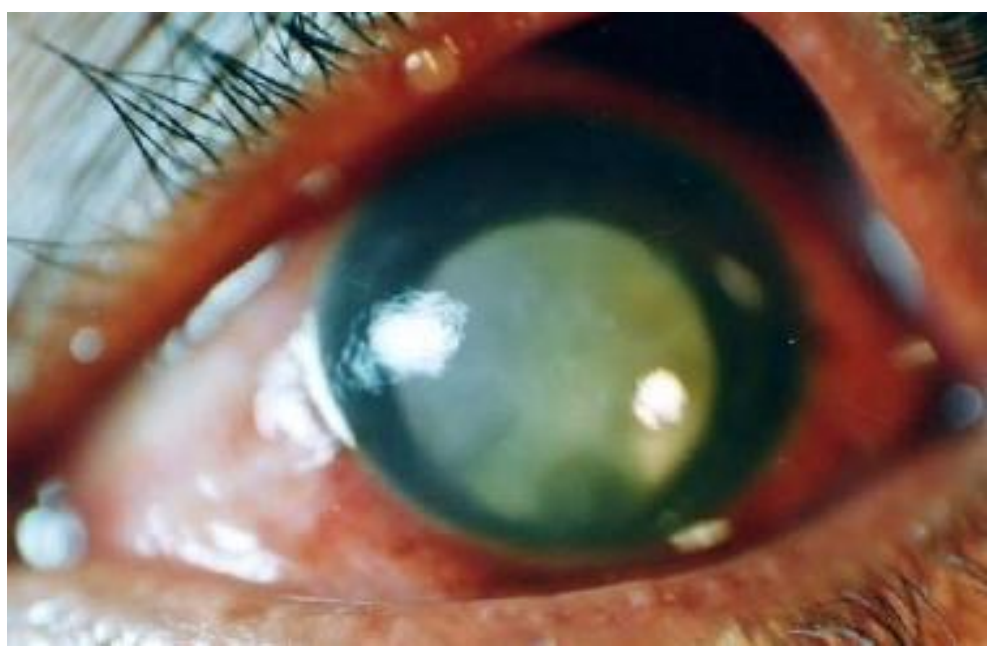

FIGURE 5: PRE-OPERATIVE IOP IN VARIOUS LIG

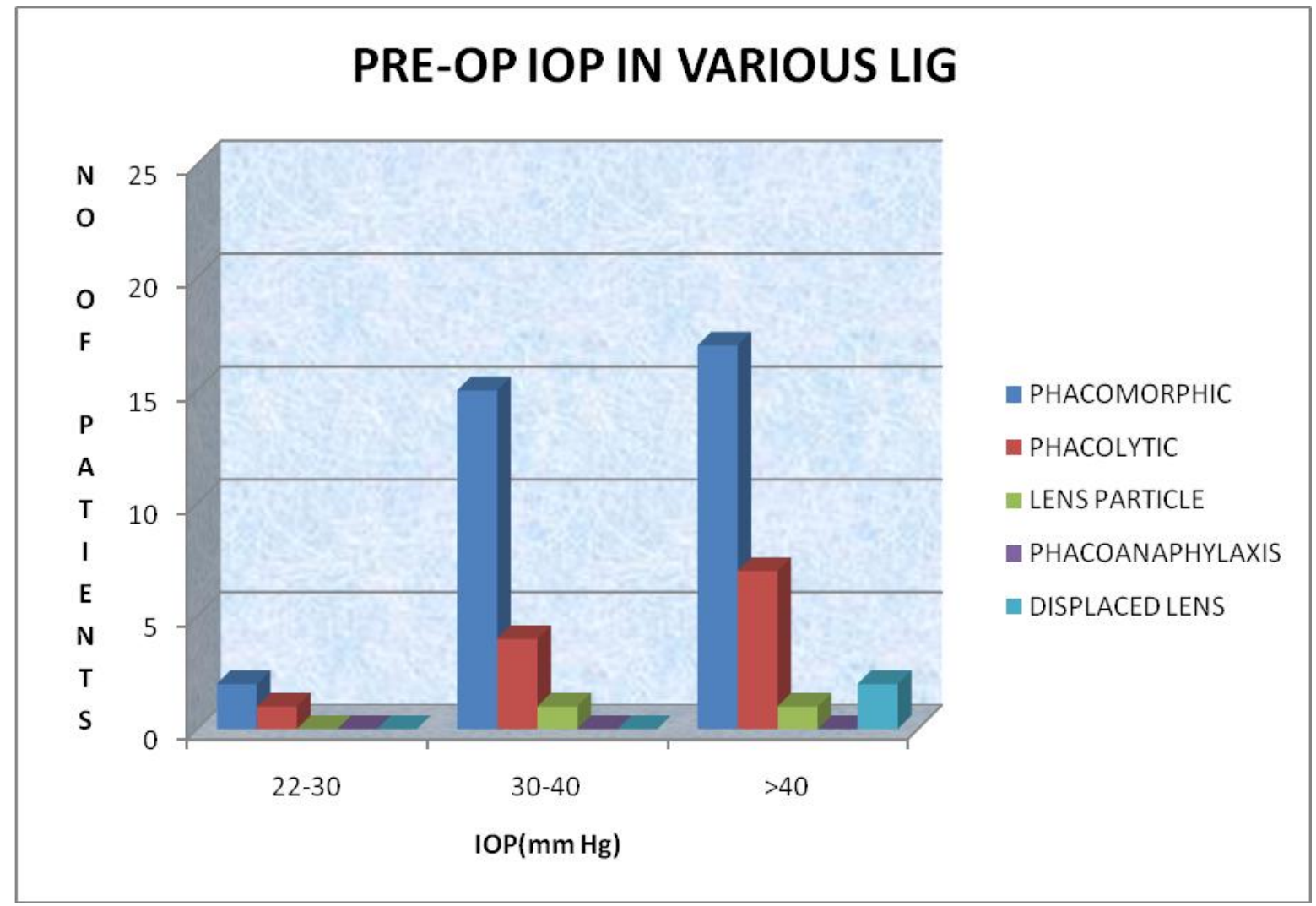




\section{ORIGINAL ARTICLE}

FIGURE 6: PRE-OP IOP AND FINAL BCVA

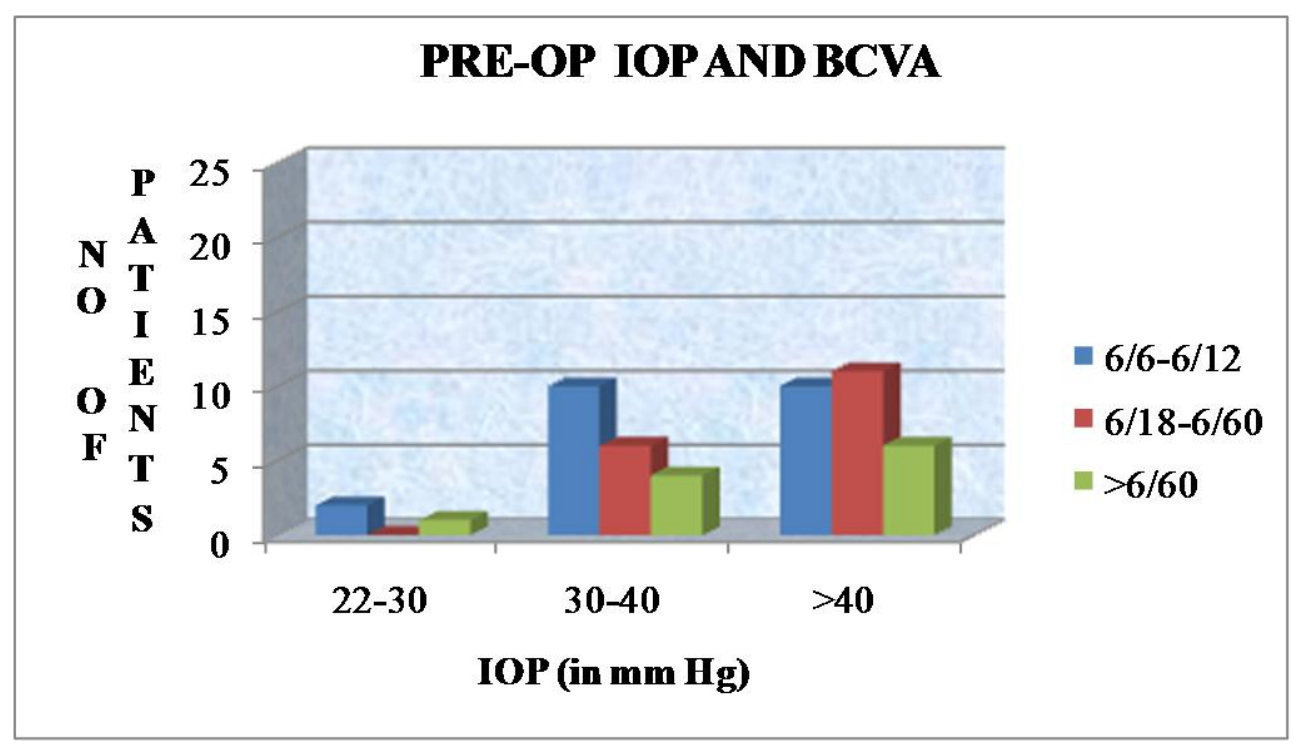

